\title{
LOS MODALIZADORES DE ATENUACIÓN COMO (QUE), IGUAL, MEDIO/A, DE REPENTE Y CAPAZ (QUE) EN EL HABLA SANTIAGUINA: ANÁLISIS PRAGMÁTICO Y SOCIOLINGÜÍSTICO ATTENUATION MODAL MARKERS COMO (QUE), IGUAL, MEDIO/A, DE REPENTE AND CAPAZ (QUE) IN SANTIAGO, CHILE'S SPEECH: PRAGMATIC AND SOCIOLINGUISTIC ANALYSIS
}

\author{
Jorge Sandoval Cárcamo \\ Universidad de Chile \\ jorge.sandoval.c@ug.uchile.cl \\ Abelardo San Martín Núñez \\ Universidad de Chile \\ asmartin@uchile.cl
}

\section{RESUMEN}

En este trabajo se analiza el comportamiento pragmático y la estratificación sociolingüística de los modalizadores de atenuación: como (que), igual, medio/a, de repente y capaz (que) en el habla santiaguina. Específicamente, se abordan estas partículas como recursos de la función pragmático-discursiva de modalización atenuadora, en relación con el tipo de género discursivo y con su posición sintácticopragmática preferente, así como la correlación de su empleo con los factores sociodemográficos: sexo-género, edad y grupo socioeconómico de los hablantes. Los principales hallazgos muestran que el modalizador de atenuación como (que) presenta la mayor frecuencia de uso en la muestra, mientras que la edad es el factor sociodemográfico más sensible al empleo de este tipo de partículas.

Palabras clave: partícula discursa, modalización, atenuación, sociolingüística, español de Chile.

\begin{abstract}
This paper analyzes the pragmatic behavior and the sociolinguistic stratification of the attenuation modalities: como (que), igual, medio/a, de repente, and capaz (que) in Santiago's speech. Specifically, these particles are approached as resources of the pragmatic-discursive function of attenuating modality, in relation to the type of discursive gender and its preferred syntactic-pragmatic position, as well as the correlation of its employment with the sociodemographic factors: sex-gender, age and socioeconomic group of speakers. The main findings show that the attenuation modality como (que) has the highest frequency of use in the sample, while age is the sociodemographic factor most sensitive to the use of this type of particles.
\end{abstract}

Keywords: discursive particle, modalization, attenuation, sociolinguistics, Chilean Spanish.

Recibido: 05 de mayo de 2020

Aceptado: 10 de junio de 2020 
Artículo. Jorge Sandoval Cárcamo y Abelardo San Martín Núñez. "Los modalizadores de atenuación como (que), igual, medio/a, de repente y capaz (que) en el habla santiaguina: análisis pragmático y sociolingüístico".

\section{Introducción}

Una de las funciones (entre otras) desde la que se han analizado las partículas del discurso es la función de modalización atenuadora. Esta última, siguiendo a autores como Briz (La estrategia) y Albelda y Cestero, desempeña un papel primordial en la dimensión interaccional y se concibe como una estrategia o recurso que permite "quitar relieve, mitigar, suavizar, restar fuerza elocutiva, reparar, esconder la verdadera intención” (Briz, 2003). La modalización, en tanto estrategia discursiva general, participa en la esfera de la interacción comunicativa entre los interlocutores, quienes con frecuencia efectúan modalizaciones (como la de atenuar) en un sentido comunicacional estratégico, mediante las cuales reflejan su actitud (modus) ante el contenido de lo dicho (dictum). Diversos trabajos han estudiado cuáles son aquellas partículas del discurso que cumplen la función de modalización atenuadora en el español de España y de América, a partir, principalmente, de estudios con orientación semasiológica (cf. Montes, Briz (El español), Rojas, Holmvik, Albelda y Cestero, Jørgensen, Kornfeld, Panussis y San Martín, Mondaca, entre otros). Los resultados generales de estos trabajos muestran la importancia del empleo recurrente de como (que), además de de repente e igual, en tanto modalizadores de atenuación en español. El objetivo general de este trabajo es analizar las partículas como (que), igual, mediola, de repente y capaz (que) como recursos variantes de la función de modalización atenuadora en una muestra de 72 entrevistas sociolingüísticas representativas del habla de Santiago de Chile; específicamente, nos proponemos determinar su frecuencia de uso y su comportamiento pragmático, además de establecer la distribución sociolingüística de su empleo en correlación con las variables sociales sexo-género, edad y grupo socioeconómico de los sujetos. Nuestras hipótesis son, en primer lugar, que la partícula de modalización atenuadora como (que) es la que presenta la mayor frecuencia de uso y, en segundo término, que la edad del sujeto es el factor socioeconómico más sensible al empleo de las partículas de modalización atenuadora seleccionadas en la muestra. 
Nueva Revista del Pacífico 2020, No 72, (pp. 145-172). ISSN (e) 0719-5176

\section{Marco Teórico}

Los modalizadores del discurso responden a un tipo específico de partículas que, según la categorización funcional de Briz et al., proyectan la actitud del hablante hacia un estado mental que se desea comunicar ya sea de manera intensificada o atenuada. En específico, aquí nos interesan los modalizadores de atenuación que constituyen una estrategia de distanciamiento lingüístico y de acercamiento social (Briz, 2003), en la cual el contenido del mensaje se mitiga, mientras que se produce un acercamiento en la relación interpersonal de los interlocutores; por lo tanto, la atenuación se relaciona con la estrategia de cortesía verbal, ya que el interlocutor se aleja del enunciado a la vez que se acerca en su relación interpersonal con los demás. Sin embargo, aunque el empleo de la atenuación pragmática puede estar asociado a la cortesía, no toda imprecisión en el mensaje se asocia directamente con las relaciones personales entre hablantes, sino que puede existir solo una aproximación del contenido semántico motivado por un mero conocimiento incompleto o parcial de lo dicho. De este modo, existen algunos recursos que en español permiten expresar "la información como no exacta, pero cercana a la verdad, los que revelan un modo de hablar voluntariamente impreciso, o basado en comparaciones o acercamientos” (Fuentes 226). Esta aproximación semántica, aunque se realiza de manera intencional, no siempre está ligada a la cortesía verbal, puesto que en su empleo no se involucran las imágenes públicas de los interlocutores (Goffman). Pareciera ser que los hablantes en la comunicación realizan una búsqueda de la expresión semántica adecuada y, al no encontrarla, deciden el uso atenuado del contenido informativo, a fin de salvaguardar la máxima de calidad informativa de Grice.

Con todo, no toda expresión "borrosa" del enunciado se realiza de forma alejada del contexto y los interlocutores. La atenuación lingüística, como ya señalamos, puede ligarse no solo a las intenciones del hablante, sino que también a la relación interpersonal entre los interlocutores; de esta forma, funciona en un sentido estratégico ligado a la cortesía verbal para salvaguardar las imágenes públicas de los interlocutores. En palabras de Briz (Notas 67), la atenuación puede funcionar como “a) mecanismo de minimización de las acciones

del yo hablante; b) mecanismo que minimiza una posible amenaza a la imagen o a los derechos del otro; y c) mecanismo reparador de las acciones que han perjudicado la imagen o han ocupado el territorio del otro". Este tipo de función ha sido estudiada, en lo principal, 
Artículo. Jorge Sandoval Cárcamo y Abelardo San Martín Núñez. "Los modalizadores de atenuación como (que), igual, medio/a, de repente y capaz (que) en el habla santiaguina: análisis pragmático y sociolingüístico".

como fórmula estratégica mediante recursos lingüísticos atenuadores como los diminutivos, las oraciones interrogativas y las partículas discursivas como de repente ("tal vez" o “quizás”) (cf. Briz, La atenuación, El español, La estrategia, Notas; Puga, Montecinos, Albelda y Cestero).

Por otro lado, se ha demostrado que la función pragmática que los interlocutores le otorgan a ciertas partículas se ve influida tanto por sus posibilidades de combinación, como por su posición en el enunciado (Briz y Pons). En particular, en el caso de los modalizadores de atenuación se ubican, de modo prototípico, en posición inicial de acto o subacto siendo mínima su presencia en posición final lo que se debe, quizás, a la naturaleza morfosintáctica principal de los atenuantes: adjetivos, adverbios y locuciones adjetivas y adverbiales. Ejemplos de esto pueden ser bueno (Briz, Notas) y como(que) (Panussis y San Martín), los que desempeñan, en general, un valor de atenuación en posición inicial mientras que modifican su función en otras posiciones enunciativas. Además, es frecuente que las fórmulas atenuativas se combinen en el discurso formando verdaderos "bloques atenuativos", esto es, una secuencia de atenuadores, demostrando una amplia capacidad de combinación.

Con respecto al estudio de las partículas que aquí analizamos, Puga y Montecino abordan la atenuación en muestras de español chileno, destacando el empleo de partículas como igual, un poco y como, mediante las cuales "el hablante no se responsabiliza de aplicar el contenido de su predicado en toda su intensidad semántica” (Montecino 23). San Martín (Igual), por su parte, estudia el valor de igual como reformulador de distanciamiento el que, en ciertos contextos, asumiría también un valor atenuador. Rojas, asimismo, señala que la partícula de repente, más allá de su significado adverbial ("súbitamente”), puede emplearse con el sentido de "a veces" o "tal vez", significados que, si bien siguen siendo intraoracionales (afectan al verbo), traen consigo una reducción de la fuerza ilocutiva y argumentativa del enunciado, con un efecto atenuador. Panussis y San Martín y Mondaca, además, indagan las funciones aproximadora y atenuadora de como (que) en el español chileno. Sobre la incidencia de factores sociodemográficos de los hablantes en el empleo de partículas atenuadoras, Albelda y Cestero, en muestras de hablantes de Valencia y Madrid, señalan que la atenuación es mayoritariamente juvenil, aunque para el caso de las partículas atenuadoras, en específico, la tercera edad es la que toma ventaja en dichas comunidades de 
habla peninsular. Por su parte, Panussis y San Martín señalan que en Santiago de Chile el uso de como (que) con función atenuadora lo emplean más los hablantes jóvenes. Por supuesto, esto refleja diferencias dialectales del español valenciano y madrileño con el santiaguino que sería interesante indagar en términos geo y sociolingüísticos.

\section{Metodología}

\subsection{Corpus}

El corpus que sirvió de base para nuestro estudio corresponde a 72 entrevistas sociolingüísticas pertenecientes al corpus del grupo de Estudio Sociolingüístico del Español de Chile (ESECH). Las entrevistas fueron realizadas a hablantes santiaguinos, entre 2005 y 2011, por los estudiantes en la asignatura de Sociolingüística de los programas de Licenciatura en Lengua y Literatura Hispánicas y Licenciatura en Lengua y Literatura Inglesas de la Universidad de Chile. En la situación de entrevista, los entrevistadores debían tratar de superar la "paradoja del observador" consiguiendo, de esta forma, una muestra significativa de discurso natural grabado (estilo vernáculo) de hablantes representativos de la comunidad de habla en estudio (Labov). Los procedimientos aplicados en la recopilación del corpus, así como las características del instrumento (entrevista semiestructurada) son descritos con detenimiento en San Martín y Guerrero. La transcripción de las entrevistas antes señaladas fue exhaustivamente revisada, sin exclusión de ninguna de las secciones del instrumento.

\subsection{Población y muestra}

En nuestra investigación se consideró la población constituida por hombres y mujeres de la Región Metropolitana de más de 20 años. El cuestionario se aplicó a una muestra por cuotas con afijación uniforme, en la que se divide a la población en estratos o categorías y se asigna una cuota a cada uno de los distintos estratos (López Morales 58). La muestra, así conformada, comprende un total de 72 entrevistas realizadas a igual número de sujetos, distribuidos como se indica en la tabla 1: 
Artículo. Jorge Sandoval Cárcamo y Abelardo San Martín Núñez. "Los modalizadores de atenuación como (que), igual, medio/a, de repente y capaz (que) en el habla santiaguina: análisis pragmático y sociolingüístico".

Tabla 1. Distribución de sujetos según características sociodemográficas de sexo, edad y grupo

\begin{tabular}{|c|c|c|c|c|c|c|c|}
\hline & \multicolumn{2}{|c|}{$20-34$} & \multicolumn{2}{c|}{$35-54$} & \multicolumn{2}{c|}{55 y más } & M \\
\hline & H & M & H & M & H & Tal \\
\hline Medio alto & 3 & 3 & 3 & 5 & 3 & 3 & $=18$ \\
\hline Medio & 3 & 3 & 3 & 5 & 3 & 3 & $=18$ \\
\hline Medio bajo & 3 & 3 & 3 & 5 & 3 & 3 & $=18$ \\
\hline Bajo & 3 & 3 & 3 & 5 & 3 & 3 & $=18$ \\
\hline & 12 & 12 & 12 & 12 & 12 & 12 & $=72$ \\
\hline
\end{tabular}

\subsection{Procedimiento de estratificación social utilizado en ESECH}

Para la estratificación de los sujetos que conforman la muestra del estudio, se empleó el sistema de adscripción de estatus social empleado en ESECH que se basa en la asignación del puntaje -que se indica entre paréntesis- a los informantes, de acuerdo con las siguientes variables: a) nivel educacional (3), b) profesión u ocupación (2) y c) comuna de residencia (1). La explicación en detalle de dichas variables se encuentra en San Martín y Guerrero, que consiste en un procedimiento de estratificación basado en determinados estudios acerca de la realidad socioeconómica chilena. A partir de la asignación de los mencionados puntajes, se definieron cuatro grupos socioeconómicos, definidos según los rangos siguientes: a) Medio alto (MA): 42 - 36, b) Medio (M): 35 - 27, c) Medio bajo (MB): 26 18 y d) Bajo (B): 17 - 6. El intervalo de cada rango responde a la mayor coincidencia con los puntajes que han establecido los estudios sociológicos y de mercado para la clasificación de estratos socioeconómicos, aunque con las debidas adaptaciones, puesto que en esta muestra no se incluyen ni el grupo de la extrema pobreza ni el de la extrema riqueza.

\subsection{Procedimiento analítico}

En nuestro análisis consideramos las frecuencias de aparición de los modalizadores de atenuación seleccionados en el corpus y la distribución de su uso atendiendo a las variables sociodemográficas consideradas en este trabajo, a saber, sexo-género, edad y grupo socioeconómico. De manera paralela, con el fin de determinar si nuestros resultados poseen o no proyección más allá de nuestra muestra, realizamos un análisis estadístico inferencial 
que incluyó una prueba paramétrica (ANOVA) y otra no paramétrica (Anova de KruskalWallis). En ambos casos, el grado de significación se definió en el 5\%, según el cual $\mathrm{p}=<0,05$ fue considerado estadísticamente significativo. El paquete estadístico al que hemos recurrido para la estadística inferencial es el SPSS (Statistical Package for the Social Sciences), versión 15.0 para Windows. Es necesario señalar que para efectos del análisis cuantitativo solo se tomaron en consideración aquellos modalizadores cuya frecuencia absoluta en el corpus fue igual o superior a 25 casos. Esta decisión se adecúa a las recomendaciones de Hernández Campoy y Almeida.

\section{Análisis de los resultados}

\subsection{Análisis pragmático}

Se registraron 3431 casos en los que las partículas discursivas seleccionadas cumplen la función pragmática de modalización atenuadora en el corpus. Las frecuencias de uso específicas de cada una se expresan en la siguiente tabla:

Tabla 2: Frecuencia absoluta y porcentajes de modalizadores de atenuación: capaz (que), de repente, medio/a, igual, como (que).

\begin{tabular}{|c|c|c|}
\hline Marcador & Frecuencia absoluta & Porcentaje \\
\hline Como (que) & 2873 & $83,74 \%$ \\
\hline Igual & 435 & $12,68 \%$ \\
\hline Medio/a & 80 & $2,33 \%$ \\
\hline De repente & 42 & $1,22 \%$ \\
\hline Capaz (que) & 1 & $0,03 \%$ \\
\hline Total & 3431 & $100 \%$ \\
\hline
\end{tabular}

Como se observa en la tabla 2, el predominio de como (que) para expresar atenuación lingüística en la muestra del habla santiaguina analizada es claro, por lo que se corrobora nuestra hipótesis referida a su uso preferente por sobre las otras partículas seleccionadas. A dicho atenuador le sigue el empleo de igual y, a mucha distancia, medio/a y de repente y, de forma casi inexistente, capaz (que). Todas estas partículas discursivas, siguiendo la clasificación de Cortés y Camacho, son de carácter interaccional y no textual puesto que 
Artículo. Jorge Sandoval Cárcamo y Abelardo San Martín Núñez. "Los modalizadores de atenuación como (que), igual, medio/a, de repente y capaz (que) en el habla santiaguina: análisis pragmático y sociolingüístico".

"tienen la función de orientar al oyente sobre las inferencias que tiene que hacer a propósito de las relaciones socioafectivas entre hablantes" (Cortés y Camacho 26). Asimismo, según nuestro análisis, estas partículas en su funcionamiento pragmático de modalización atenuadora presentan particularidades que los caracteriza como tales. De esta forma, tanto ciertas posiciones como la expresión argumentativa de opiniones de los interlocutores sobre temas controversiales motivarán, mayormente, la ocurrencia de los atenuadores. De igual manera, en muchas ocasiones los entrevistados construyen verdaderos "bloques atenuativos" en los que se presenta una secuencia de atenuadores coordinados unos con otros con un significado atenuador total. Además, aunque no en forma de bloque, pero sí presentes de modo discontinuo en una misma intervención, estos atenuadores siguen un "rastro atenuativo" que los interlocutores van construyendo en sus enunciados. Es decir, a modo general de su enunciación, los interlocutores van atenuando su discurso a medida que lo construyen dando como resultado una intervención atenuada en términos generales, lo que se relaciona con la temática de la conversación y con la postura argumentativa que adoptan los interlocutores de su propio discurso y del de los demás, expresando, en algunos casos, recursos de cortesía verbal asociada a la atenuación lingüística y, en consecuencia, como señala Briz (La estrategia, Notas), al cuidado de las imágenes de las dimensiones del discurso como, por ejemplo, el cuidado de la imagen propia (face).

\subsubsection{Como (que)}

Como (que) es, por lejos, el modalizador de atenuación más frecuente en la muestra del habla santiaguina analizada. Esta partícula, ya abordada anteriormente en Panussis y San Martín y Mondaca, cumple con la función de modalización atenuadora, por un lado, de forma semántica aproximativa, un uso no estratégico que expresaría un desconocimiento epistémico de lo que el hablante enuncia (Fuentes) y, por otro, con un fin estratégico que se liga a una finalidad de cortesía en salvaguarda de las imágenes públicas de los interlocutores (Briz, Notas). De acuerdo con el DLE (s. v. como), los significados adverbiales de cómo, “aproximadamente, o más o menos" y "atenúa el grado de certeza de lo que se expresa a continuación", se condicen con la aproximación semántico-enunciativa y con el uso estratégico ligado a la finalidad de cortesía, respectivamente. A continuación, se presentan ejemplos del significado aproximativo de como: 
1) E: ¿y en Maipú cuándo vivió?

I: estuve como dos años

E: ah / poquito (B II H017) ${ }^{1}$.

2) I: al principio // era una embarrada // o sea yo me demoraba / de mi trabajo / a mi casa / como tres horas // y tenía que / de una micro / bajarme al metro / del metro otra micro / y de ahí / otra micro más [...] (B I M011).

Como puede apreciarse en 1) y 2), el uso de como (que) lleva asociado la aproximación semántica de tal manera que el hablante aproxima y presenta borrosa la cuantificación que enuncia enseguida (“dos años" y "tres horas"). En estos casos, como no ejerce funciones intraoracionales, más bien su función es extraoracional, ligada a la marcación modalizadora del discurso. Con respecto a esto, es frecuente en nuestro corpus que este tipo de aproximación enunciativa se realice cuando el hablante o bien narra hechos pretéritos, o bien especula aproximativamente sobre el futuro, vinculándose, de esta forma, con el género discursivo narrativo. Esta situación de lejanía epistémico-temporal en la que se ubica el yo hablante, motivaría la aparición de este tipo de como (que) aproximativo, que es una señal al oyente de que no debe interpretar dicha cantidad como un número exacto, sino más bien, como uno difuso e impreciso.

A continuación, se presentan otros ejemplos de esta partícula modal:

\footnotetext{
${ }^{1}$ Respecto de la transcripción de los ejemplos, creemos necesario indicar las siguientes convenciones gráficas: 1) cuando corresponda a ejemplos de mayor extensión, $\mathrm{E}=$ entrevistador e I = informante; 2) como se trata de variables no fonéticas y, por lo tanto, la pronunciación no es el foco de nuestro análisis, el texto correspondiente a cada ejemplo se transcribe en ortografía convencional, incluidos los acentos gráficos; 3 ) con el propósito de no desnaturalizar demasiado la representación del habla chilena, se ha conservado la manifestación gráfica de determinados usos característicos del español hablado en Chile, como el uso del voseo paradigmático con elisión de $-\mathrm{s}$, por ejemplo, estái, soi, viví (estáis, sois, vivís con valor de segunda persona del singular, tú) y el empleo de marcadores del discurso, como po (pues), ¿cachái? (¿entiendes?); 4) para la mejor comprensión de los ejemplos incluidos en este estudio, se ha suprimido el sistema de etiquetas pertenecientes al Standard Generalized Markup Language (SGML) contenidas en el Text Encoding Initiative (TEI), que se emplea en la transcripción de las entrevistas de ESECH, excepto las pausas, que se señalan con I, y algunos rasgos prosódicos que ayudan a comprender el funcionamiento de los marcadores de relleno, específicamente, las pausas, los alargamientos, los énfasis y los silencios. Los marcadores analizados se presentarán siempre en letra cursiva. Al final de cada ejemplo, se indica, entre paréntesis, el código del informante, según las siguientes convenciones: grupo socioeconómico $(\mathrm{MA}=$ medio alto, $\mathrm{M}=$ medio, $\mathrm{MB}=$ medio bajo, $\mathrm{B}=$ bajo), grupo etario (III = adultos mayores de 55 años y más, $\mathrm{II}=$ sujetos adultos de edad intermedia entre 35 y 54 años y $\mathrm{I}=$ hablantes jóvenes de entre 20 y 34 años) y sexo $(\mathrm{M}=$ mujer y $\mathrm{H}=$ hombre). A continuación del sexo se señala el número correlativo del sujeto en el corpus.
} 
Artículo. Jorge Sandoval Cárcamo y Abelardo San Martín Núñez. "Los modalizadores de atenuación como (que), igual, medio/a, de repente y capaz (que) en el habla santiaguina: análisis pragmático y sociolingüístico".

3) I: no / he tenido sueños así que como que de repente me ponen mal // porque son sueños feos y despierto así como asustada o/ o como que ando todo el día con el sueño pegado en mi mente / pero premonitorio no / aparte que no creo en esas cosas (MB II M074).

4) I: cosas así y aparte que uno se siente inseguro entre tanto flaite como que a uno le da miedo un poco el miedo que le pueda pasar algo / eso / pero es como más que propiamente es como la sensación del ambiente o la atmósfera que como que a uno lo / lo inhibe un poco. (MA I H145).

Como puede apreciarse en 3) y 4), en cuanto a su posibilidad de combinación sintáctica, como (que) es un elemento muy versátil, ya que puede combinarse con formantes como el adverbio así o el verbo ser (antepuestos) o el subordinante que (pospuesto). Con su función de modalización atenuadora, como (que) permite, por un lado, la aproximación difusa del contenido enunciativo y, por otro, la posibilidad de que esta atenuación se ligue a una finalidad de cortesía. En este sentido, como puede apreciarse en 3), como (que) puede también relacionarse al cuidado de la imagen de los hablantes en "no / he tenido sueños así que como que de repente me ponen mal", ante una autovaloración del tipo bueno/malo, el hablante decide usar el atenuador como que para disminuir la fuerza argumentativa de lo dicho. Por consiguiente, I mitiga el contenido negativo del mensaje, ya que este puede poner en peligro su propia imagen, activando una labor protectora estratégica que le sirva para no exponerse como una persona débil. Al uso de como que en función de mitigación discursiva, se le suma de repente, con el sentido de "a veces", el que sirve como un recurso que también disminuye la fuerza argumentativa (Rojas). De modo similar, en 4), se puede apreciar que I utiliza de manera estratégica la atenuación linguiística en función de proteger su propia imagen. En este sentido, al opinar sobre sí mismo y exponerse como "miedoso", decide atenuar el contenido de "a uno le da miedo" y de "a uno lo inhibe". Esto se suma a la expresión un poco que, según Mariottini, también tiene un valor aproximativo.

Igualmente, consideramos que la función de introducción de cita de así + como comentada por Panussis y San Martín conlleva un efecto de sentido atenuador, puesto que la 
introducción de cita presenta de forma mitigada su contenido informativo. Esto se ejemplifica en el siguiente caso:

5) I: tú vái caminando por la calle y te topái con una conversación de flaites y escuchái algo más o menos así como "cha loco no pa' si fui pa' ya cachai y los cabros y la hueá na' no si no / no pasa na"” y / y quedái colgado poh” (MB I H051).

Como se aprecia en 5), así + como introduce una cita en discurso directo (en subrayado). Interpretamos que el hablante, al narrar los hechos y aludir a una cita de la que no se tiene total precisión de su contenido informativo, decide incluir así + como con el fin de guiar al oyente hacia una interpretación matizada o aproximada de su contenido.

La posición sintáctico-discursiva de como (que) en su función modalizadora no es azarosa, puesto que se ubica en posición inicial o intermedia y nunca en posición final de acto o subacto. Por otro lado, este modalizador de atenuación es versátil en su combinación con otras formas atenuadoras como un poco y de repente, debido, en gran medida, a su alta frecuencia, la que propicia la construcción de "bloques atenuativos" en los que varios atenuadores se yuxtaponen en un mismo enunciado:

6) I: sí trato de cambiarla pero de repente igual como que salen las palabras y me dicen “¿qué dijiste?” y se los tengo que volver a explicar (B I H001).

Como se aprecia en 6), este tipo de construcción "pero de repente igual como que" es recurrente en la muestra, lo que puede reflejar una característica del habla chilena. Dicha construcción se compone de un conector adversativo pero + el adverbio de repente + el reformulador de distanciamiento igual + un atenuador como(que). Esta fórmula de "bloque atenuativo" en un sentido adversativo y distanciado del subacto anterior es un ejemplo de aglomeración de atenuadores que se expresan como una atenuación global en bloque. De esta manera, el hablante presenta un primer subacto, a saber: "sí / trato de cambiarla (mi forma de hablar)" y, en seguida, la secuencia adversativa atenuada: "pero de repente igual como que" que funciona, en este caso, como un argumento que excusa al hablante de, en 
Artículo. Jorge Sandoval Cárcamo y Abelardo San Martín Núñez. "Los modalizadores de atenuación como (que), igual, medio/a, de repente y capaz (que) en el habla santiaguina: análisis pragmático y sociolingüístico".

definitiva, "no cambiar su forma de hablar al hablar con mayores", puesto que "las palabras salen de su boca" siendo este último, el subacto atenuado por como (que), reflejando, a su vez, la vinculación de esta partícula con el género discursivo argumentativo.

\subsubsection{Igual}

Igual es el segundo modalizador de atenuación en frecuencia de uso. A continuación, se presentan dos ejemplos de esta partícula:

7) I: yo le aconsejo / hija no tome mi amor // y no < vacilación>/ no me hace caso / yo la aconsejo / igual / de repente se me desbanda / esa es la oveja negra que tengo / y está a mi lado // porque sin mí ella no es nada // y tengo mi maravillosa nietas nietos / tengo tres / cinco nietos (MB III M091).

8) I: cómo carreteo / en general// mucho carrete en casa/ en general como que me gusta más más relajado también no// no soy de salir a perrear ni nada aunque igual perreo a ratos// y generalmente eso como el carrete de casa o irse a un/ a un bar <alargamiento/> // como esa clase de cosas (MA I H147).

Como se aprecia en 7) y 8), igual no funciona en su sentido oracional de adjetivo o adverbio de igualdad (DLE s. v. igual), sino que, más bien, ejerce un rol más allá de la oración, una función discursiva de reformulación de distanciamiento ya analizada por San Martín (Igual, Los reformuladores). Esta función de reformulación de distanciamiento argumentativo de igual genera un efecto de sentido en segundo plano ligado a la atenuación. En consecuencia, en 7), el hablante presenta un primer acto, a saber: "yo la aconsejo" lo que a continuación se reformula con "igual de repente" y, luego, un nuevo acto que será el determinante por encima del anterior: "se me desbanda". De esta forma, igual en estos casos presenta dos posibilidades: una anterior que se invalida y otra posterior que se destaca como la pertinente. Este procedimiento es el que genera un efecto de sentido ligado a la atenuación en la que, en definitiva, el contenido del mensaje se presenta 
aproximado mediante la alusión a dos posibilidades lo que, además en el ejemplo 7, se ve mayormente atenuado con la combinación con de repente, en el sentido de "habitualidad esporádica". De forma similar, en 8), se presenta un primer acto: "no soy de salir a perrear ni nada" al que le sigue el conector concesivo aunque y el reformulador igual para señalar un segundo acto: "perreo a ratos", esto genera la alusión a dos caminos posibles, lo que, en definitiva, es una guía inferencial que direcciona hacia una interpretación imprecisa, borrosa, en la que el oyente deberá interpretar si es que I realmente "no baila" o si es que "baila a ratos". Esta distancia discursiva en la que se propone un nuevo argumento como el verdaderamente determinante, en contraposición a los anteriores, no se liga necesariamente con la atenuación con fines de cortesía señalada por Briz (Notas), ya que, en realidad, expresa una duda epistémica que el hablante tiene y que intenta corregir con la reformulación, según la aproximación semántica no estratégica señalada en Fuentes, siendo, más bien, la reformulación en sí, como un acto argumentativo, la que podría justificarse en términos de cortesía verbal. En cuanto a su posición sintáctico-discursiva, igual como reformulador con efecto de sentido atenuativo con mayor frecuencia toma una posición inicial de acto o subacto como se observa en 8), donde aparece en posición inicial del segundo acto "igual perreo a ratos". En síntesis, igual refleja en primera instancia una función de reformulación de distanciamiento la que, además, puede llevar a un efecto de sentido ligado a la atenuación que se refuerza con su combinación con otras partículas atenuadoras como de repente y como (que).

\subsubsection{Medio/a}

Medio/a presenta la tercera posición de frecuencia en el corpus. Según el DUECH (s. v. medio), se trata de un adjetivo cuyo significado modifica "una característica en un grado no muy alto ni muy bajo" permitiendo a los hablantes mitigar o quitar relieve al contenido enunciativo de manera estratégica, ligado, como hemos visto, a un fin de cortesía verbal. A continuación, presentamos ejemplos que ilustran este uso:

9) E.: ¿por qué no sabes si era hombre o mujer?

I.: porque nunca se le vio polola todos decían que era medio mariconcito / la verdad es que nunca vi un <vacilación/> / algo que lo delatara de ese 
Artículo. Jorge Sandoval Cárcamo y Abelardo San Martín Núñez. "Los modalizadores de atenuación como (que), igual, medio/a, de repente y capaz (que) en el habla santiaguina: análisis pragmático y sociolingüístico".

modo/ pero era como un personaje extraño dentro del curso (MB II M073).

10) I: y quise cambiarme a la línea uno/ y / y en vez de salir al/ a la línea uno/ salí a la calle entonces tuve que <ininteligible/> es que yo soy medio despistado/ entonces/ no cacho mucho los trasbordos y esas cuestiones (MA III H178).

Como se aprecia en 9) y en 10), medio/a, sintácticamente, siempre precede a otro adjetivo (en los ejemplos, mariconcito y despistado). En este sentido, en 9) se aprecia cómo, ante la temática controversial "sexualidad", I atenúa, empleando medio/a y el sufijo -ito, el contenido de maricón con una finalidad posiblemente ligada a la cortesía, puesto que el hablante, al quitar el relieve argumentativo, se distancia del contenido de su discurso para proteger su propia imagen ante la amenaza del empleo de un término discriminatorio. Con este tipo de atenuación, el hablante a medida que pone borroso el contenido de su enunciado distanciándose de él, se acerca o salvaguarda la relación interpersonal con el interlocutor (labor preventiva), esto ocurre, sobre todo, en casos en los que la temática es controversial. De modo semejante, en 10) vemos la protección de la propia imagen de I al momento de atenuar el contenido despistado que, como adjetivo calificativo, puede causar cierto daño a su propia imagen (autocalificación negativa). En este sentido, el género argumentativo se liga de forma directa con la función de atenuación linguiística efectuada por este marcador, en tanto sirve al hablante como un recurso para expresar opiniones o argumentos controversiales de forma no totalmente comprometida. En cuanto a su posición, esta partícula se ubica, preferentemente, en posición inicial o intermedia modificando a un adjetivo.

\subsubsection{De repente}

La partícula discursiva de repente aparece en penúltimo lugar de frecuencia en la muestra analizada. Como señala Rojas y DUECH, posee tres significados posibles, a saber: 1) loc. adv. con significado oracional "de modo súbito", 2) loc. adv. "con frecuencia esporádica” y 3) marcador discursivo, "quizá, tal vez que se usa para indicar que lo que se dirá 
continuación debe interpretarse como algo probable pero incierto para el hablante" (DUECH s. v. de repente). Para este estudio será pertinente este último significado, el que se ejemplifica a continuación:

11) I: a mí me gustan mucho los amigos // mucho mucho los amigos/ igual me gustaría atender <énfasis> mucha </énfasis> gente en mi casa atender a muchas personas <ininteligible/> pero de repente igual a veces uno tiene mala suerte con los amigos también / porque no todos los amigos son / buena onda (B II M025).

Como puede apreciarse en 11), este modalizador de atenuación mitiga o reduce la fuerza del contenido enunciado por el hablante de manera tal que guía al oyente hacia una interpretación aproximada de los mismos. I presenta un primer acto: "me gustaría atender mucha gente en mi casa atender a muchas personas" y mediante el conector adversativo pero condiciona el hecho de que "no la visiten amigos" y que, por tanto, "no pueda atender a muchos de ellos en su casa". Por consiguiente, el segundo acto "uno tiene mala suerte con los amigos" se presenta como la causa de que el primer acto no se realice. Esta causa es la que el hablante decide atenuar de una manera estratégica, ya que, siguiendo a Briz (Notas), I pondría un "escudo protector" a su propia imagen al momento de mitigar "uno tiene mala suerte con los amigos porque no todos los amigos son / buena onda" puesto que ésta es una opinión que podría poner en riesgo su imagen. En este sentido, de repente es usado en forma "preventiva". Esta construcción sintáctico-discursiva del tipo "adversativo (pero) + atenuador (de repente) + reformulador de distanciamiento (igual)" es recurrente en el uso de los modalizadores de atenuación, en general. Asimismo, la posición de este atenuador es la inicial de subacto o acto, lo que responde a una tendencia general de las partículas atenuadoras.

\subsubsection{Capaz (que)}

Capaz (que) cumple la función de modalizador de atenuación solo en una oportunidad en el corpus. Según el DUECH (s. v. capaz) "se usa para que lo que se dirá a continuación deba interpretarse como algo probable pero incierto para el hablante"; por lo tanto, capaz (que) representa un recurso de atenuación. Sin embargo, este modalizador tiene escasa ocurrencia 
Artículo. Jorge Sandoval Cárcamo y Abelardo San Martín Núñez. "Los modalizadores de atenuación como (que), igual, medio/a, de repente y capaz (que) en el habla santiaguina: análisis pragmático y sociolingüístico".

y, siguiendo a Heine, representa un estadio inicial o intermedio de gramaticalización, ya que coocurren ambos usos, a saber, el de significado intraoracional que es más prevalente y el de partícula discursiva, esto es, en posición sintáctica periférica (extraoracional) y separado entre comas. El único ejemplo de capaz como partícula discursiva que se documentó es el siguiente:

12) I: $\leq$ tipo = argumentativo > yo pienso que los que se tiene de niño / capaz / son más valederos porque son / con la conciencia limpia / sin sin / llegar a a tener o tratar de obtener ganancia con su amistad / en cambio de adulto los amigos entran a tallar muchos intereses / hay amistad / eh / que solamente son interesadas en algo </tipo =argumentativo > (M III H131).

En 12), a la vez que el I se aleja del contenido de su mensaje dejándolo borroso, se preocupa por el cuidado de la imagen propia y del oyente, de modo que presenta de manera aproximada o mitigada el contenido "las amistades de la infancia son las mejores", puesto que no queda totalmente claro si esa es su opinión definitiva. Así, capaz sirve como un recurso lingüístico para expresar dicha vacilación o poco compromiso con el propio discurso. Los tipos de cortesía que asociamos a este ejemplo, siguiendo a Briz (Notas), son el cuidado de la autoimagen y el cuidado de la imagen del interlocutor.

\subsection{Análisis sociolingüístico}

Como ya se mencionó en 3.1., las pruebas de estadística inferencial se aplicaron a las partículas con más de 25 ocurrencias (cf. tabla 2); por lo tanto, hemos excluido a capaz. (que) de este procedimiento, debido a su escasísima presencia en el corpus. Cabe destacar, además, que los datos se distribuyen de forma anormal en la muestra puesto que la distribución del empleo de las partículas atenuadoras entre los sujetos es bastante desigual, debido a esto se reforzó el análisis paramétrico con pruebas no paramétricas (cf. 3.1.).

\subsubsection{Sexo-género}

La tabla 3 presenta la frecuencia de empleo de las partículas de modalización atenuadora analizadas, según el sexo-género de los hablantes: 
Tabla 3: Frecuencia absoluta y porcentaje de los modalizadores de atenuación relevados en el corpus según el sexo de los sujetos de la muestra

\begin{tabular}{|c|c|c|}
\cline { 2 - 3 } \multicolumn{1}{c|}{} & \multicolumn{2}{c|}{ Sexo-género } \\
\hline Modalizador & Hombres & Mujeres \\
\hline Como (que) & $1346(46,85 \%)$ & $1527(53,15 \%)$ \\
\hline Igual & $193(44,37 \%)$ & $242(55,63 \%)$ \\
\hline Medio/a & $49(61,25 \%)$ & $31(38,75 \%)$ \\
\hline De repente & $9(21,43 \%)$ & $33(78,57 \%)$ \\
\hline Total & $1598(46,59 \%)$ & $1832(53,41 \%)$ \\
\hline
\end{tabular}

Como se aprecia en la tabla 3, hombres y mujeres emplean los modalizadores de atenuación analizados de forma variada; en total, las mujeres utilizaron más los modalizadores de atenuación que los hombres. En consecuencia, los datos arrojan una leve inclinación porcentual hacia las mujeres por sobre los hombres en el uso de estos modalizadores de atenuación, a excepción de medio/a. Desde un punto de vista estadístico inferencial, por otra parte, la media de los hombres fue 34,46 y la de las mujeres 38,54; no obstante, los resultados de ANOVA fueron $\mathrm{F}=0,681$ y $\mathrm{p}=0,413$ y los de Kruskal-Wallis, Chi cuadrado= 0,686 y p= 0,408, por lo que las diferencias en el uso de los modalizadores de atenuación relevados considerados entre hombres y mujeres no son significativas estadísticamente. En cuanto a como (que), el atenuador más empleado, también se verifica un empleo preferente en mujeres, lo que concuerda con lo señalado en Panussis y San Martín y que se relaciona con lo que al respecto indica Blas Arroyo (167): “[las mujeres] tienden a atenuar en mayor medida que los hombres. En el habla de éstos, sin embargo, los actos de habla mitigadores son mucho menos frecuentes". Sin embargo, como ya señalamos, desde el punto de vista inferencial, la aplicación de las pruebas ANOVA y Kruskal-Wallis no avala esta asociación entre el uso de estas partículas atenuadoras y el discurso femenino. 
Artículo. Jorge Sandoval Cárcamo y Abelardo San Martín Núñez. "Los modalizadores de atenuación como (que), igual, medio/a, de repente y capaz (que) en el habla santiaguina: análisis pragmático y sociolingüístico".

\subsubsection{Edad}

En la tabla 4 se presenta la frecuencia de uso de las partículas seleccionadas, de acuerdo con la edad de los sujetos de la muestra:

Tabla 4: Frecuencia absoluta y porcentaje de los modalizadores de atenuación relevados en el corpus según la edad de los sujetos de la muestra

\begin{tabular}{|c|c|c|c|}
\cline { 2 - 4 } \multicolumn{1}{c|}{} & \multicolumn{3}{c|}{ Grupo etario } \\
\hline Modalizador & $20-34$ & $35-54$ & $55 \mathrm{y}+$ \\
\hline Como (que) & $1337(46,54 \%)$ & $1059(36,86 \%)$ & $477(16,60 \%)$ \\
\hline Igual & $221(50,81 \%)$ & $139(31,95 \%)$ & $75(17,24 \%)$ \\
\hline Medio/a & $35(43,75 \%)$ & $30(37,5 \%)$ & $15(18,75 \%)$ \\
\hline De repente & $9(21,43 \%)$ & $13(30,95 \%)$ & $20(47,62 \%)$ \\
\hline Total & $1602(46,691 \%)$ & $1241(36,17 \%)$ & $588(17,137 \%)$ \\
\hline
\end{tabular}

Porcentualmente, cerca de la mitad de los casos registrados se concentra en el grupo más joven de la muestra. De modo consecuente, además, el empleo de modalizadores de atenuación va disminuyendo a medida que aumenta la edad de los sujetos, a excepción de de repente que manifiesta un comportamiento inverso a esta tendencia. Este patrón decreciente es bastante claro en las frecuencias de como (que) e igual con función atenuadora. Resultados similares se registran en Jørgensen, Panussis y San Martín y San Martín (Igual, Los reformuladores). Nuestros hallazgos relativos al uso preferentemente juvenil de este tipo de modalizadores en el español santiaguino, sin embargo, no coinciden con los de Albelda y Cestero quienes, con respecto a las partículas de atenuación en el habla de Madrid y Valencia, señalan que su empleo es mayor en informantes de la tercera edad. Esta discrepancia, por un lado, refuerza la idea del factor etario como una variable sociodemográfica relevante $y$, por otro, destaca la existencia de variación interdialectal que resulta de notable interés. Para nosotros, el uso más juvenil de los modalizadores de atenuación se puede explicar debido una "non-committal stance" (Andersen), con respecto al propio discurso que es más recurrente en jóvenes que en etapas etarias posteriores, debido, posiblemente, a inseguridad discursiva o a un proceso aun incompleto de construcción en el discurso de una identidad propia. Estos aspectos, quizás, podrían motivar 
Nueva Revista del Pacífico 2020, No 72, (pp. 145-172). ISSN (e) 0719-5176

la aparición más frecuente de modalizadores de atenuación en los jóvenes, puesto que funcionan como mitigadores que matizan la fuerza argumentativa de sus enunciados.

Los resultados de ANOVA $(\mathrm{F}=10,097 ; \mathrm{p}=0,000)$ y de Kruskal-Wallis (Chi cuadrado= 16,480; $\mathrm{p}=0,000)$ comprueban la significación estadística de este factor, en términos inferenciales. Las medias correspondientes son 66,792 (20-34); 51,708 (35-54) y 24,458 (55 y más años), evidenciando un patrón decreciente no abrupto en el comportamiento de este tipo de partículas en relación con la variable etaria (ver gráfico 1). Por lo tanto, verificamos una mayor predisposición en el grupo juvenil hacia la modalización atenuativa del discurso y, en consecuencia, al uso de los modalizadores de atenuación aquí analizados.

Gráfico 1: Medias marginales estimadas del total del empleo de las partículas de modalización atenuadora consideradas según la edad de los sujetos

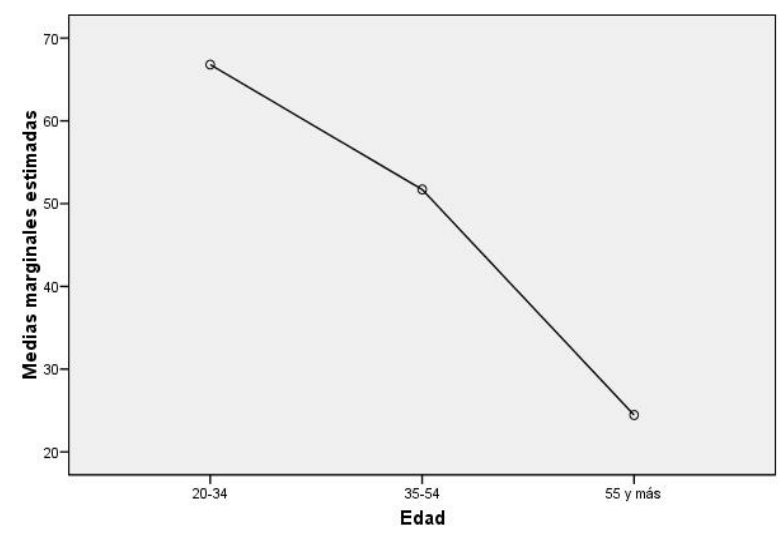

Por lo que refiere al comportamiento de modalizadores de atenuación específicos, destacamos los siguientes casos:

1) El contraste de medias de como (que) arroja los siguientes valores: 55,708 (20-34), 44,125 (35-54) y 19,875 (55 y más años), según un patrón también decreciente (ver gráfico 2). La prueba paramétrica $(\mathrm{F}=8,810 ; \mathrm{p}=0,000)$ y la no paramétrica (Chi cuadrado $=15,106 ; \mathrm{p}=0,001)$ confirman la significatividad estadística de esta asociación entre como (que) en su función atenuadora y los hablantes más jóvenes (cf. Panussis y San Martín).

Gráfico 2: Medias marginales estimadas del empleo de como (que) atenuador según la edad de los sujetos 
Artículo. Jorge Sandoval Cárcamo y Abelardo San Martín Núñez. "Los modalizadores de atenuación como (que), igual, medio/a, de repente y capaz (que) en el habla santiaguina: análisis pragmático y sociolingüístico".

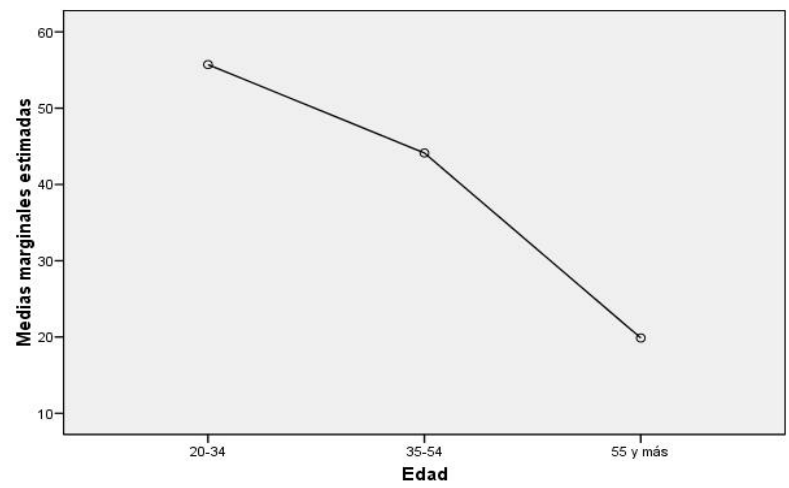

2) La varianza de igual con valor atenuador indica las siguientes medias: 9,208 (2034), 5,792 (35-54) y 3,125 (55 y más años), representando un patrón decreciente que se diferencia del empleo de como (que) y del total de marcadores, en que la media del grupo etario intermedio en el caso de igual es menor (ver gráfico 3). Tanto ANOVA $(\mathrm{F}=5,413 ; \mathrm{p}=0,007)$ como Kruskal-Wallis (Chi cuadrado= 12,099; $\mathrm{p}=0,002$ ) avalan la significación estadística de la asociación entre igual y los hablantes de la edad más joven (cf. San Martín Los reformuladores).

Gráfico 3: Medias marginales estimadas del empleo de igual atenuador según la edad de los sujetos

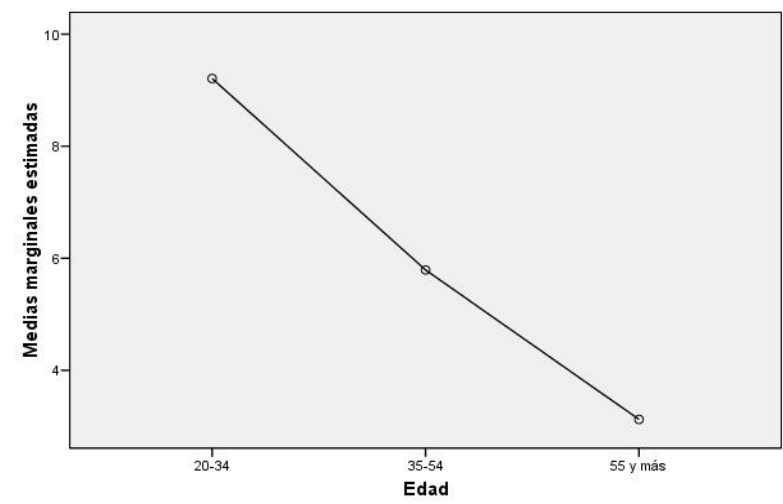

De esta forma, la variable edad fue la más sensible al empleo de los modalizadores de atenuación por lo que se verifica la hipótesis de este estudio al respecto.

\subsubsection{Grupo socioeconómico}

La tabla 5 detalla la frecuencia del empleo de los modalizadores de atenuación considerados, de acuerdo con el grupo socioeconómico de los hablantes: 
Nueva Revista del Pacífico 2020, No 72, (pp. 145-172). ISSN (e) 0719-5176

Tabla 5: Frecuencia absoluta y porcentaje de los modalizadores de atenuación relevados en el corpus según el grupo socioeconómico de los sujetos de la muestra

\begin{tabular}{|c|c|c|c|c|}
\cline { 2 - 5 } \multicolumn{1}{c|}{} & \multicolumn{4}{c|}{ Grupo socioeconómico } \\
\hline Modalizador & B & MB & M & MA \\
\hline Como (que) & $563(19,60 \%)$ & $760(26,45 \%)$ & $696(24,23 \%)$ & $854(29,72 \%)$ \\
\hline Igual & $90(20,69 \%)$ & $164(37,70 \%)$ & $122(28,05 \%)$ & $59(13,56 \%)$ \\
\hline Medio/a & $22(27,50 \%)$ & $18(22,50 \%)$ & $15(18,75 \%)$ & $25(31,25 \%)$ \\
\hline De repente & $9(21,43 \%)$ & $12(28,57 \%)$ & $14(33,33 \%)$ & $7(16,67 \%)$ \\
\hline Total & $684(19,936 \%)$ & $954(27,805 \%)$ & $848(24,726 \%)$ & $945(27,543 \%)$ \\
\hline
\end{tabular}

Según el grupo socioeconómico de los hablantes, en términos descriptivos, se verifica un uso más frecuente de los modalizadores de atenuación en los grupos medio bajo y medio alto de la escala. De forma similar, el modalizador de atenuación más frecuente de la muestra, como (que), refleja un empleo levemente mayor de los grupos medio bajo y medio alto por sobre el grupo bajo y medio siguiendo la tendencia del total. En cambio, igual como modalizador de atenuación manifiesta un comportamiento diferente: su empleo es mayor en los grupos medio bajo y medio y menor en el grupo medio alto y bajo. El contraste de las medias es el siguiente: 31,67 (Bajo); 41,03 (Medio bajo); 35,22 (Medio) y 38,08 (Medio alto). Sin embargo, las pruebas de ANOVA $(F=0,775$ y $p=0,514)$ y de Kruskall-Wallis (Chi cuadrado=1,974 y p=0,578) no verifican la significación estadística de estas diferencias de empleo. Por lo tanto, a nuestra consideración, la asociación entre el empleo de los modalizadores de atenuación con el factor sociodemográfico de grupo socioeconómico se trata solo de una posibilidad que debería corroborarse con una muestra mayor.

\subsubsection{Intersección entre variables}

Las únicas dos intersecciones que dieron un resultado significativo fueron el cruce entre la edad y el grupo socioeconómico del sujeto para el caso de medio/a (ANOVA: F= 2,434; p= 0,036) e igual con valor atenuador (ANOVA: $\mathrm{F}=3,660 ; \mathrm{p}=0,004)$. Con respecto a medio/a, la varianza fue: I) 20-34: Bajo (1,500), Medio Bajo (1,167), Medio (0,333), Medio Alto (2,833); II) 35-54: Bajo (1,667), Medio Bajo (1,333), Medio (1,000), Medio Alto (1,000); III) 55 y más años: Bajo (0,500), Medio Bajo (0,500), Medio (1,167), Medio Alto $(0,333)$ (ver gráfico 4). 
Artículo. Jorge Sandoval Cárcamo y Abelardo San Martín Núñez. "Los modalizadores de atenuación como (que), igual, medio/a, de repente y capaz (que) en el habla santiaguina: análisis pragmático y sociolingüístico".

Gráfico 4: Medias marginales estimadas de medio/a según el cruce de edad y grupo socioeconómico del sujeto

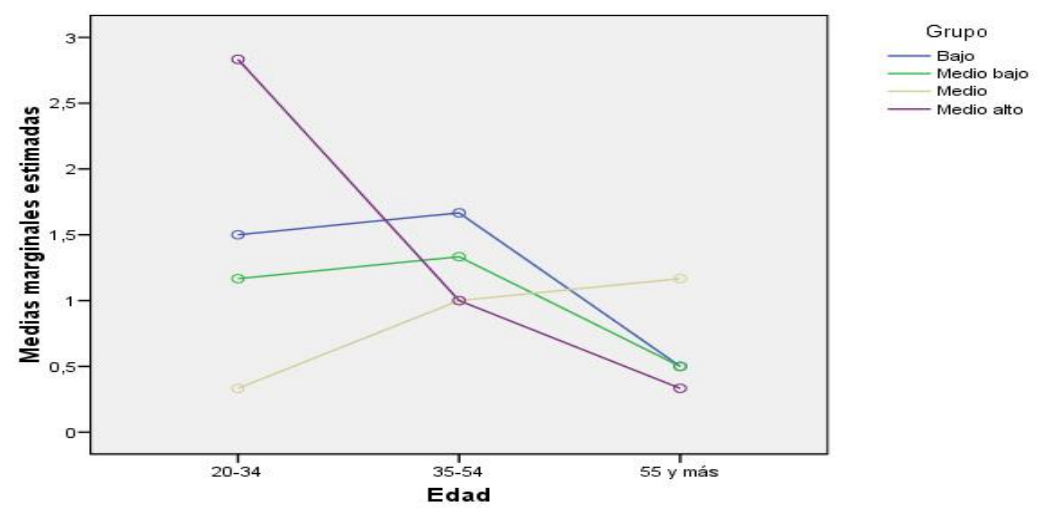

Como se observa, los sujetos del grupo etario I (20-34 años) y del grupo socioeconómico Medio Alto emplean en mayor medida medio/a con función de modalización atenuadora. Esta situación cambia de forma abrupta y significativa con el avance de la edad; al alcanzar el grupo etario II (35-54 años), el patrón de comportamiento decae para, ya en el grupo etario III (55 y más años), llegar a su punto más bajo. En consecuencia, el grupo etario III (55 y más años) presenta la media más baja. Sin embargo, en el caso del grupo socioeconómico Medio, este patrón se invierte: el grupo de la tercera edad y del grupo Medio emplea más este modalizador con función atenuadora.

Por su parte, la varianza de igual con valor atenuador es: I) 20-34: Bajo (5,500), Medio Bajo (19,500), Medio (7,667), Medio Alto (4,167); II) 35-54: Bajo (7,667), Medio Bajo $(5,167)$, Medio (7,167), Medio Alto $(3,167)$; III) 55 y más años: Bajo $(1,833)$, Medio Bajo $(2,667)$, Medio (5,500), Medio Alto (2,500) (ver gráfico 5). 
Gráfico 5: Medias marginales estimadas de igual según el cruce de edad y grupo socioeconómico del sujeto

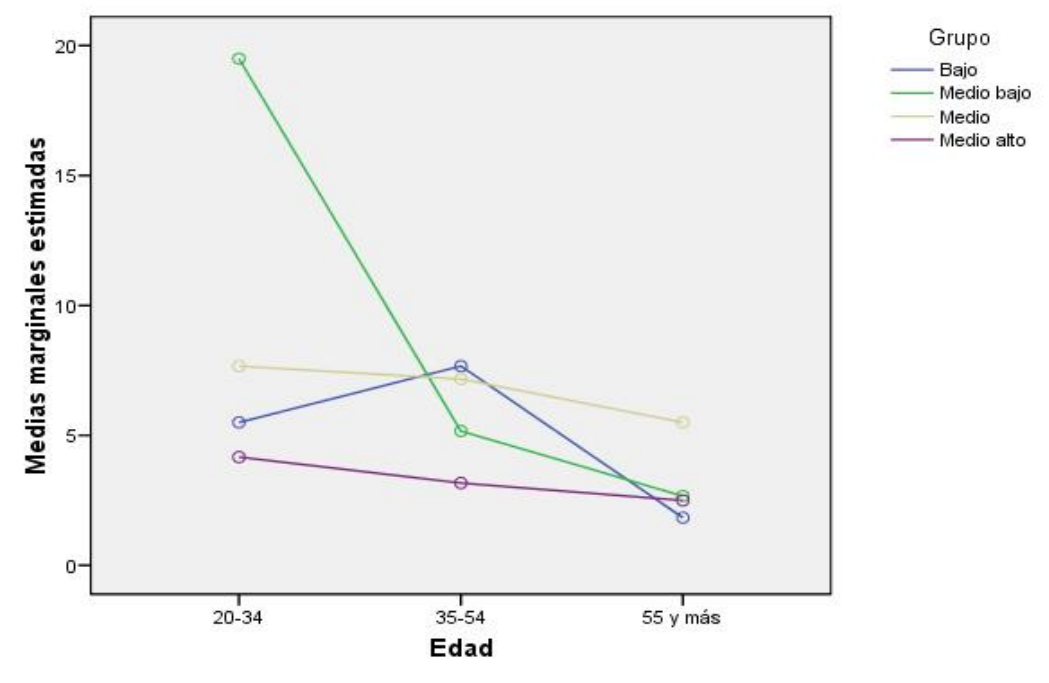

Como se ve, los sujetos del grupo etario I (20-34 años) y del grupo socioeconómico Medio Bajo emplean en mayor medida igual con función atenuadora. Esta situación cambia conforme avanza la edad del sujeto; al alcanzar el grupo etario II (35-54 años), el patrón de comportamiento decae abruptamente para, ya en el grupo etario III (55 y más años), llegar a su punto más bajo. En el caso del grupo Bajo, el empleo es preferentemente en el grupo etario intermedio. Finalmente, el comportamiento de los grupos Medio y Medio Alto no ofrece diferencias relevantes. 
Artículo. Jorge Sandoval Cárcamo y Abelardo San Martín Núñez. "Los modalizadores de atenuación como (que), igual, medio/a, de repente y capaz (que) en el habla santiaguina: análisis pragmático y sociolingüístico".

\section{Conclusiones}

Desde un punto de vista pragmático, como(que), igual, medio/a, de repente y capaz(que) son partículas discursivas que funcionan como modalizadores de atenuación en el habla santiaguina. Como (que) presenta la mayor frecuencia de uso en la muestra analizada y se combina, además, de manera muy recurrente con otros recursos atenuadores. Con respecto a su posición sintáctico-discursiva, estos modalizadores se ubican con mayor frecuencia en posición inicial de acto o subacto previamente al contenido que atenúan (Albelda y Cestero). Esta situación, muy probablemente se debe a su base gramatical adverbial u adjetival, la que motivaría su anteposición a otros constituyentes discursivos. Asimismo, las temáticas en las que aparecen, en general, pueden ser contenidos controversiales en los que no exista una sola opinión o postura. Por este motivo, los modalizadores de atenuación se vinculan, sobre todo, con el tipo de género argumentativo o de opinión, puesto que sirven al hablante como recursos de atenuación de la fuerza argumentativa de sus aseveraciones. En consecuencia, esta estrategia les permite un posicionamiento matizado en el que no se expresa un compromiso total con el propio discurso. De esta forma, la atenuación lingüística, en general, como una herramienta estratégica y las partículas de modalización atenuadora, en específico, pueden ser explicadas por medio de la cortesía verbal, puesto que se relacionan con el cuidado de las imágenes públicas de los interlocutores (Briz, Notas). Por último, de un lado, la yuxtaposición consecutiva de modalizadores de atenuación, como se viene comentando, genera un efecto de "bloque atenuativo", muy frecuente en nuestros materiales del español santiaguino, que puede estar unido a otras funciones discursivas como la reformulación de distanciamiento (por ejemplo, el empleo de igual) y la adversatividad, mientras que, de otro, el uso de modalizadores de atenuación previos genera un "rastro atenuativo" que puede dar un sentido mitigado general al discurso. De esta forma, este tipo de marcadores no solo funcionan en un nivel de acto o subacto, sino que también pueden afectar a niveles superiores del discurso.

Desde el punto de vista sociolingüístico, hemos verificado que la edad es el factor sociodemográfico más sensible al empleo de los modalizadores de atenuación relevados. De esta forma, los jóvenes utilizan una mayor cantidad de estas partículas que el resto de los hablantes de los demás grupos etarios, formando un patrón descendente. Es posible que 
esto se deba a condicionamientos relacionados con la expresión de una mayor inseguridad discursiva propia del grupo juvenil, de acuerdo con Jørgensen, Andersen y Panussis y San Martín. La incidencia de este factor es evidente en el empleo de como (que), que es la partícula predominante para desempeñar la función de modalización atenuadora y que es, también, más frecuente entre los hablantes más jóvenes. En cuanto a la variable sexogénero, hemos evidenciado que esta no supone grandes diferencias, aunque, levemente, el empleo de estas partículas es preferentemente femenino. Por su parte, en cuanto al factor grupo socioeconómico, tampoco relevamos diferencias significativas. Asimismo, la intersección entre las variables edad y grupo socioeconómico arrojó significatividad estadística en el caso de las partículas medio/a e igual. Al respecto, medio/a es empleado mayormente por jóvenes del grupo socioeconómico Medio Alto, mientras que igual lo es por parte de jóvenes del grupo socioeconómico Medio Bajo. Estos resultados nos permiten confirmar nuestras hipótesis, a saber, a) como (que) es el modalizador de atenuación de mayor empleo en la muestra y b) la variable etaria es el factor sociodemográfico más sensible al empleo de los modalizadores de atenuación en Santiago de Chile.

Por último, cabe destacar que, además de los modalizadores de atenuación aquí considerados, existen otros que pueden expresar atenuación lingüística y que pueden ajustarse al paradigma de las partículas discursivas, por ejemplo, un poco, tal vez, quizás, casi (que), entre otros adjetivos, adverbios y locuciones que expresan modalidad epistémica y mitigación en el contenido enunciativo y que sirven de guía para una interpretación atenuada o de no compromiso del hablante con respecto al contenido enunciado. Por lo tanto, sería interesante extender esta perspectiva analítica al estudio de otras partículas modalizadoras de atenuación, así como validar a futuro nuestros resultados sobre los modalizadores aquí analizados. Con este artículo hemos pretendido contribuir al estudio de esta dimensión del uso del español en Chile, en particular.

*Este estudio forma parte del proyecto FONDECYT Regular N¹161422, “Los marcadores del discurso en el español hablado en Santiago de Chile: análisis pragmático y sociolingüístico". Se sintetizan, reformulan y amplían aquí los principales hallazgos de la tesis de licenciatura de Jorge Sandoval Cárcamo, “Análisis pragmático y sociolingüístico de modalizadores de atenuación en el habla de Santiago de Chile: como (que), igual, medio/a, de repente, y capaz (que)", investigación dirigida por Abelardo San Martín Núñez, en el marco del proyecto antes mencionado. 
Artículo. Jorge Sandoval Cárcamo y Abelardo San Martín Núñez. "Los modalizadores de atenuación como (que), igual, medio/a, de repente y capaz (que) en el habla santiaguina: análisis pragmático y sociolingüístico".

\section{Referencias}

Academia Chilena de la Lengua. Diccionario de uso del español de Chile. Santiago de Chile: MN Editorial, 2010.

Albelda, Marta y Ana María Cestero. "La atenuación lingüística como fenómeno variable." La lengua, lugar de encuentro. Actas XVI Congreso Internacional de la ALFAL. Eds. Ana María Cestero, Isabel Molina y Florentino Paredes. Alcalá: Servicio de Publicaciones de la Universidad de Alcalá, 2012. 1857-1866.

Andersen, Gisle Pragmatic Markers and Sociolinguistic Variation. A Relevance-theoretic Approach to the Language of Adolescents. Amsterdam: John Benjamins, 2001.

Blas Arroyo, José Luis. Sociolingüística del español. Desarrollos y perspectivas en el estudio de la lengua española en contexto social. Madrid: Cátedra, 2005.

Briz, Antonio. "La atenuación en la conversación coloquial. Una categoría pragmática”. El español coloquial. Actas del I Simposio sobre análisis del discurso oral. Ed. Luis Cortés. Almería: Servicio de Publicaciones de la Universidad de Almería, 1995. 103-122.

. El español coloquial en la conversación. Esbozo de pragmagramática. Barcelona: Ariel, 1998.

"La estrategia atenuadora en la conversación cotidiana española ". Actas del primer coloquio del programa EDICE. Ed. Diana Bravo. Estocolmo: Universidad de Estocolmo, 2003. 17-46.

"Notas para el estudio de la relación entre las partículas discursivas y la atenuación". Estudios sobre lengua, sociedad y cultura. Homenaje a Diana Bravo. Eds. María Bernal y Nieves Hernández Flores. Estocolmo: Universidad de Estocolmo [en línea]. Disponible en: http://uu.diva-portal.org/smash/get/diva2:235240/FULLTEXT01 [Consultado 01/07/2018], 2009. 73-83.

Briz, Antonio, Salvador Pons y José Portolés (coords.). Diccionario de partículas discursivas del español [en línea]. Disponible en: http://www.dpde.es [Consultado 28/06/2018], 2008.

Briz, Antonio y Salvador Pons. "Unidades, marcadores discursivos y posición”. Los estudios sobre marcadores del discurso en español, hoy. Eds. Óscar Loureda, y Esperanza Acín. Madrid: Arco/Libros, 2010. 327-358.

Cortés, Luis y María Camacho. Unidades de segmentación y marcadores del discurso. Madrid: Arco/Libros, 2005.

Holmvik, Lise. “Como” usado como marcador del discurso en el lenguaje juvenil de Madrid. Tesis Universidad de Bergen. Bergen: Universidad de Bergen, 2011. 
Nueva Revista del Pacífico 2020, No 72, (pp. 145-172). ISSN (e) 0719-5176

Fuentes Rodríguez, Catalina. “La aproximación enunciativa”. Lingüística Española Actual 30, 2 (2008): 223-258.

Goffman, Erving. La presentación de la persona en la vida cotidiana. Buenos Aires:

Amorrurtu, 1959 [2009].

Grice, Herbert Paul. "Logic and Conversation". Syntax and Semantics, Vol. 3, Speech Acts. Eds. Peter Cole y Jerry L. Morgan. New York: Academic Press, 1975. 41-58.

Heine, Bernd. "Grammaticalization”. The Handbook of Historical Linguistics. Eds. Brian D. Joseph y Richard D. Janda. Oxford: Blackwell, 2003. 573-601.

Hernández Campoy, Juan Manuel y Manuel Almeida. Metodología de la investigación sociolingüística. Málaga: Editorial Comares, 2005.

Jørgensen, Annette. "Funciones del marcador pragmático como en el lenguaje juvenil español y chileno". Pragmática y comunicación intercultural en el mundo hispanohablante. Eds. María Elena Placencia y Carmen García Fernández. London: Rodopi. 2012. 209-230.

Kornfeld, Laura Malena. “Atenuadores en la lengua coloquial argentina”. Lingüística 29 (2013): 17-49.

Labov, William. Modelos sociolingüísticos. Madrid: Cátedra, 1983.

López Morales, Humberto. Métodos de investigación lingüística. Salamanca: Ediciones Colegio de España, 1994.

Mariottini, Laura. "Modalidad y atenuación. Análisis de 'un poco' y de sus alteraciones morfológicas en las conversaciones coloquiales”. Oralia 15 (2012): 107-203.

Mondaca Becerra, Lissette. "Aproximadores y atenuadores en el español de Chile: el caso de como y como que”. Textos en Proceso 5, 1 (2019): 29-52.

Montes, José Joaquín. “Sobre el 'como’ de atenuación”. Boletín de Filología, Tomo XXXI (1980-1981): 667-675.

Montecino, Lesmer. Estrategias de intensificación y de Atenuación en la conversación coloquial de jóvenes chilenos. Onomázein 10, 2 (2004): 9-32.

Panussis, Constanza y Abelardo San Martín. “Como (que) y sus funciones discursivas en el habla santiaguina: análisis pragmático y sociolingüístico". Revista de Lingüística Teórica y Aplicada 55, 2 (2017): 30-61.

Puga, Juana. La atenuación en el castellano de Chile: un enfoque pragmalingüístico. Valencia: Tirant lo Blanch, 1997. 
Artículo. Jorge Sandoval Cárcamo y Abelardo San Martín Núñez. "Los modalizadores de atenuación como (que), igual, medio/a, de repente y capaz (que) en el habla santiaguina: análisis pragmático y sociolingüístico".

Real Academia Española. Diccionario de la lengua española. [en línea]. Disponible en: http://www.rae.es/rae.html [Consultado 28/06/2018], 2001= DLE.

Rojas, Darío. “Funciones actuales y evolución semántica de la locución 'de repente' en el español de Chile”. Boletín de Filología XLIII (2008): 207-237.

San Martín, Abelardo. "Igual como marcador discursivo en el habla de Santiago de Chile: función pragmático-discursiva y estratificación social de su empleo". Boletín de Filología XL (2004-2005): 201-232.

"Los reformuladores de distanciamiento en el habla santiaguina: igual y sus equivalentes funcionales". Onomázein 34 (2016): 261-277.

San Martín, Abelardo y Silvana Guerrero. "Estudio sociolingüístico del español de Chile (ESECH): recogida y estratificación del corpus de Santiago". Boletín de Filología L,1 (2015): 221-247. 